\section{Endovascular Correction in Acute Bleeding after Femoropopliteal Bypass: A Single-Centre Experience}

\section{Abstract}

Introduction: The Endovascular Correction (EC) has emerged in recent years as a possible alternative to surgical revision (SR) in case of bleeding complications after Peripheral Bypass (PB). The purpose of this study is to evaluate the efficacy and safety of EC compared to SR in case of bleeding complications of PB.

Methods: From January 2004 to December 2014, we have undergone surgery 32 patients for acute bleeding in previous PB (25 venous bypass, in situ or reversed, 6 PTFE bypass and 1 composite bypass). The cause of the bleeding was in 14 cases the dehiscence of proximal anastomosis, in 11 cases of distal anastomosis and in 7 cases the rupture of the graft. All patients postoperatively underwent anticoagulation or antiplatelet therapy, antibiotic therapy and close ultrasound follow-up. Patency rate, Limb Salvage rate and Survival rate in the short term were assessed by Kaplan-Meier curves.

Results: Of the 32 patients treated for acute bleeding in previous $\mathrm{PB}, 11$ underwent endovascular correction with a technical success of $100 \%$ (no need for reintervention), while in 21 patients was performed a surgical revision. Among the latter, in 4 cases (19.1\%) an early bleeding occurred, and endovascular correction was promptly performed solving the bleeding in the absence of sequelae (then a total of 15 patients were treated by endovascular correction). The 12-months patency, limb salvage and survival rates were respectively $71.4 \%, 88.2 \%$ and $88.2 \%$ in the SR group and $80 \%, 93.3 \%$ and $100 \%$ in the EC.

Conclusion: This topic underlines that endovascular correction after peripheral bypass bleeding is an attractive alternative to surgery, but there are still few data to change the gold standard treatment. Randomized Clinical Trials comparing the two treatment options are warranted.

Keywords: Endovascular correction/repair; Surgical repair; Peripheral bypass; Bleeding; Peripheral artery disease; Femoropopliteal segment; Superficial femoral artery; Popliteal artery; Comparative study

\section{Francesco Setacci, Giulia Mazzitelli, Umberto Ruzzi, Giuseppe Galzerano, Gianmarco de Donato, Domenico Benevento and Carlo Setacci}

\author{
Department of Medicine, Vascular and \\ Endovascular Surgery Unit, Surgery and \\ Neurological Sciences, University of Siena, \\ Italy
}

Corresponding author: Francesco Setacci

झ setacci@unisi.it

Department of Medicine, Vascular and Endovascular Surgery Unit, Surgery and Neurological Sciences, University of Siena, Italy.

Tel: +390577585127

Citation: Setacci F, Mazzitelli G, Ruzzi U, et al. Endovascular Correction in Acute Bleeding after Femoropopliteal Bypass: A Single-Centre Experience. J Vasc Endovasc Surg. 2017, 2:1.

Received: December 20, 2016; Accepted: January 10, 2017; Published: January 16, 2017

\section{Introduction}

Critical Limb Ischemia (CLI) represents the final stage of Peripheral Arterial Disease (PAD), in which the macro-vascular lesions induce such a reduction of the distal perfusion pressure that microcirculation and transport of nutrients are severely altered. The definition of critical ischemia has evolved over time, from the initial document of 1991 (Second European Meeting
Consensus document on CLI) to TASC I and II. Clinically is defined as a "persistently recurring ischemic rest pain requiring regular adequate analgesia for more than two weeks with an ankle systolic pressure $\leq 50 \mathrm{mmHg}$ and/or toe systolic pressure $\leq 30 \mathrm{mmHg}$; ulceration or gangrene of the foot or toes, with an ankle systolic pressure $\leq 50 \mathrm{mmHg}$ or toe systolic pressure $\leq 30 \mathrm{mmHg}$ [1]". The real incidence of critical limb ischemia is 500-1000 cases per year/ million [2]. CLI is a very severe clinical condition, with a high risk 
of major amputation, disability and death. At its presentation, 20$25 \%$ of patients are subjected to primary amputation, $50-60 \%$ are subjected to revascularization (surgical and/or endovascular) and $25 \%$ are subjected to medical treatment. At one year $20-25 \%$ of patients died, $25-30 \%$ underwent major amputation, $20 \%$ still will present critical ischemia, and only $25 \%$ of subjects will be healthy (free of signs and symptoms and without having undergone major amputation [1-4]. Atherosclerosis is the main cause of $\mathrm{PAD}$, other risk factors are: smoke, diabetes, hypertension, hypercholesterolemia and hyperhomocysteinemia [5]. Medical treatment of these risk factors is therefore essential both for primary prevention and for recurrence prevention of this disease.

Introduction of endovascular procedure shook up the concept of CLI treatment. The increasing experience of Vascular Surgeons in endovascular technique forced this specialist to match classic surgical skills with endovascular tools in order to achieve the best treatment for each patient.

In recent years the number of peripheral artery revascularization has steadily increased. This increase has inevitably resulted in a relative raise of complications. Possible complications after packaging of a peripheral bypass are mainly due to anastomosis dehiscence (anastomosis's tension, infection or rupture of the suture wire) or to a graft failure (traumatic, infective or iatrogenic), resulting often in an acute bleeding. This complication, although not very frequent, requires an immediate management to avoid sequelae which are often irreversible and potentially fatal.

The Endovascular Correction (EC) has emerged in recent years as a possible alternative to surgical revision (SR) in case of bleeding complications after Peripheral Bypass (PB). The purpose of this study is to compare the efficacy and safety of these two techniques. To minimize the risks related to these procedures, it's important that these were performed by skilled operators in high-volume centers.

\section{Materials and Methods}

From January 2004 to December 2014, we have undergone surgery 32 patients for acute bleeding in previous Peripheral Bypass (PB) (25 venous bypass, in situ or reversed, 6 PTFE bypass and 1 composite bypass). All bypass had been packaged more than 30 days before the hemorrhagic event, in different centers. The cause of the bleeding was in 14 cases the dehiscence of proximal anastomosis, in 11 cases of distal anastomosis, and in 7 cases the rupture of the graft.

\section{Pre-operative test}

All patients underwent clinical examination, $\mathrm{ABI}$ (ankle-brachial index) measurement [2-16], ultrasound examination and CTangiography scan.

\section{Intraoperative management}

Endovascular correction: An angiography was performed the beginning of the procedure in order to accurately map the femoropopliteal lesions and thus optimize the endovascular correction strategy. All patients were treated by a vascular surgeon in an operating theatre equipped with a portable fluoroscopy unit (GEOEC 9800/GE-OEC 9009; GE Medical Systems, Salt Lake City, UT, USA). Overall 15 patients underwent endovascular correction for acute bleeding. In all these patients endovascular exclusion was performed by positioning of a Viabahn Endoprosthesis ${ }^{\circledR}$ (W.L. Gore \& Associates, Newark, DE, USA) (Mean diameter $7 \mathrm{~mm}$, length from 50 to $150 \mathrm{~mm}$ ). Our choice fell on Viabahn Endoprosthesis ${ }^{\circledR}$ as the treatment of the Superficial Femoral Artery (SFA) require a flexible stent-graft capable of re-lining tortuous arteries and conform closely to the complex anatomy of the artery. In our opinion this device seems to have the most appropriate features.

Surgical repair: In these cases we paid particular attention to respect the anatomy of the surgical access, to avoid overhandling of lymph node packages (when the review of proximal anastomosis required a surgical inguinal access), to drain the hematoma as much as possible and to perform all the possible maneuvers to reduce the risk of intraoperative infection.

Post-operative management: All patients underwent anticoagulation or antiplatelet therapy, antibiotic therapy and close ultrasound follow-up (1, 3, 6, 12 months).

\section{Statistical analysis}

The Kaplan Meier method was used to show the trend in the two groups. The log rank test was used in order to detect any statistically significant difference between the two curves. Significance level was set at $\mathrm{P}<0.05$. Stata ${ }^{\circledR} \mathrm{SE}$, version 12.1 , StataCorp, College Station, Texas, USA software was used for the analysis. We report mortality rates, patency and limb salvage at 12 months in both groups of patients.

\section{Results}

Of the 32 patients treated for acute bleeding in previous PB, 11 underwent endovascular correction (Figures 1 and 2) with a technical success of $100 \%$ (no need for re-intervention), while in 21 patients was performed a surgical revision (Figure 3). Among the latter, in 4 cases (19.1\%) an early bleeding occurred, and endovascular correction was promptly performed solving the bleeding in the absence of sequelae (then a total of 15 patients were treated by endovascular correction).

\section{Surgical repair}

Among the 21 initial patients, considering the 4 underwent early endovascular correction plus 2 patients experiencing graft failure (one patient after six months for obstruction and infection of PTFE bypass (Figure 4) and another after 8 months for acute limb ischaemia with occlusion of distal anastomosis on tibio-peroneal trunk, both hesitated in amputation) we have a patency rate at 12 months of $71.4 \%$ (15 of 21 ). As regards the limb salvage rate and the survival rate at 12 months we have considered the remaining 17 patients (the 4 patients undergoing endovascular correction were subsequently considered in that group), obtaining a limb salvage rate of $88.2 \%$ (15 of 17 ) and a survival rate of $88.2 \%$ (15 of 17). As already mentioned, the two patients who underwent amputation are the same of the graft failure: one at 6 months for infection of the bypass, which necessitated explant and 


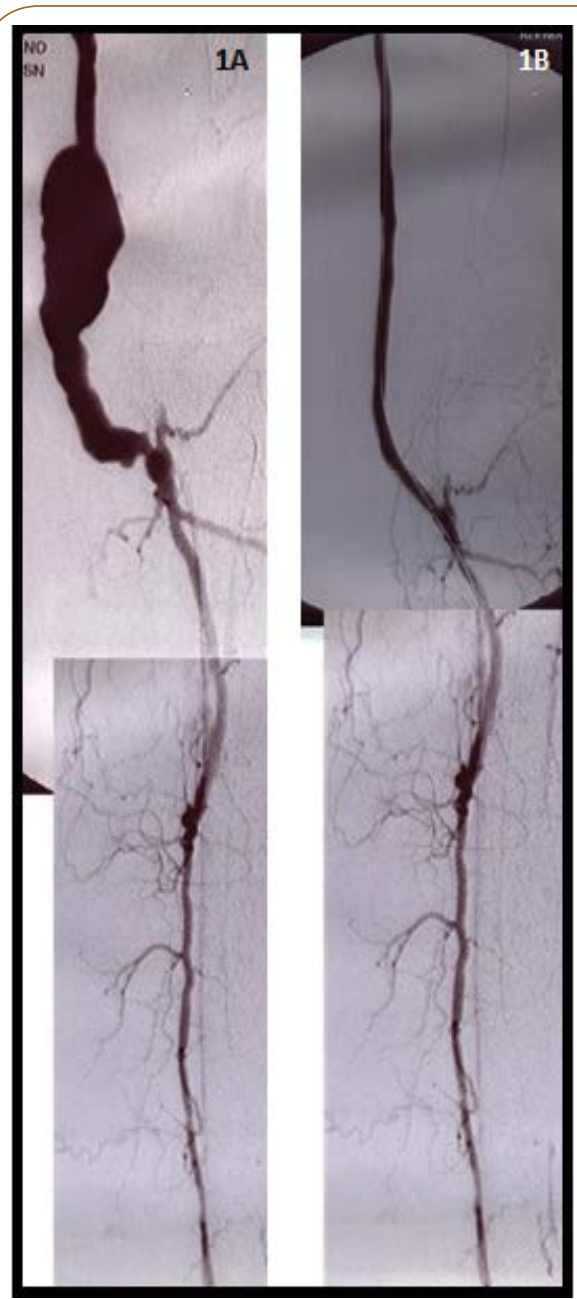

Figure 1 Endovascular correction of acute bleeding in previous composite bypass. 1A: Pre-operative digital subtraction angiography; 1B: Post-operative results.

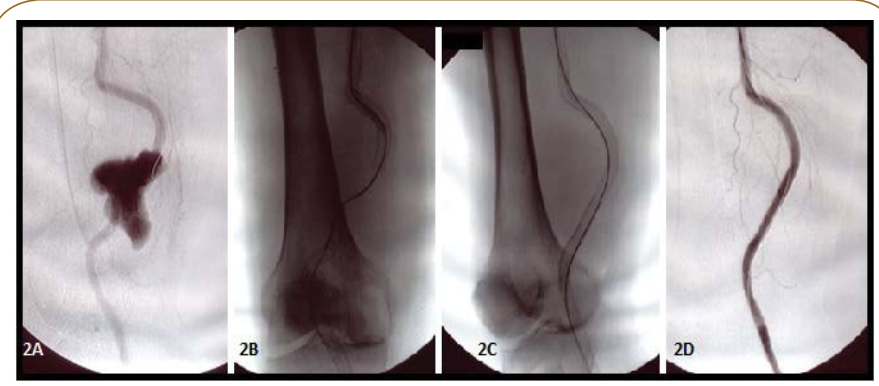

Figure 2 Endovascular correction of acute bleeding in previous endobypass for popliteal artery aneurysm exclusion. 2A: Pre-operative digital subtraction angiography; 2B: Relining of endoprosthesis; 2C: Deployment of new endoprosthesis; 2D: Post-operative results.

major amputation, and the other patient at 8 months for acute limb ischemia from distal embolization in patients with atrial fibrillation who had been subjected to fibrinolysis. Despite this, the worsening led to the amputation of his leg. The latter patient also died during hospitalization for stroke, probably also by embolization, while another patient died at 11 months for acute myocardial infarction.

\section{Endovascular correction}

Among the 15 patients treated endovascularly ( 11 primitively and 4 after failure of surgical repair) the patency rate at 12 months was $80 \%$ ( 12 of 15). Of the 3 patients experiencing graft failure 1 underwent fibrinolysis for acute occlusion of an endobypass, with subsequent clinical worsening and amputation of the leg (Limb salvage rate: 14 of $15,93.3 \%$ ), while in 2 patients was observed at 6 and 12 months the occlusion of a Viabahn ${ }^{\circledR}$ positioned in the superficial femoral artery with rehabitation of the popliteal artery by collateral circulation. They have not been subjected to reintervention for poor symptomatology. There were no deaths in this group, with a survival rate at 12 months of $100 \%$.

Summarizing the 12 -months patency, limb salvage and survival rates were respectively $71.4 \%, 88.2 \%$ and $88.2 \%$ in the SR group and $80 \%, 93.3 \%$ and $100 \%$ in the EC (Figures 5-7).

\section{Discussion}

The overall management of patients with CLI has changed radically in recent years thanks to the development of new imaging techniques that allowed an early diagnosis and setting of medical treatment in a growing percentage of cases. The purpose

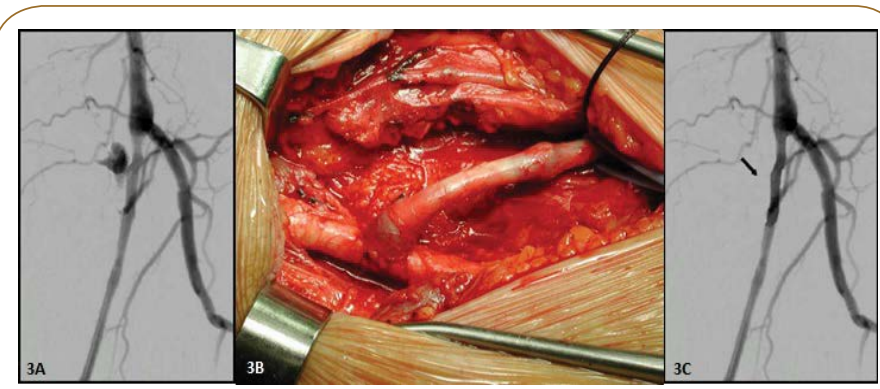

Figure 3 Surgical revision of proximal anastomosis failure in previous venous femoro-popliteal bypass. 3A: Preoperative digital subtraction angiography; 3B: Repackaging of proximal anastomosis; 3C: Post-operative results.

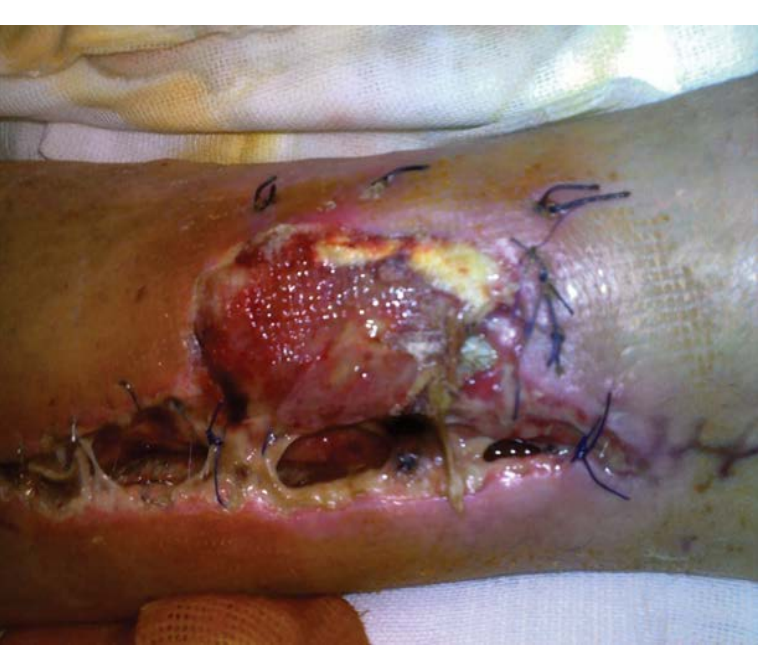

Figure 4 Infection of previous PTFE bypass. 


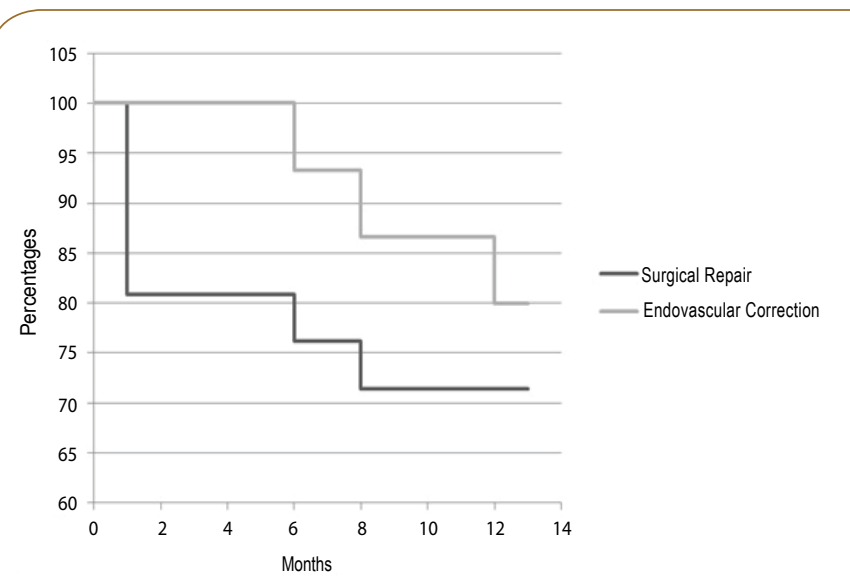

Figure 5 Graft patency rate. A comparison of graft patency rate between surgical revision and endovascular correction groups.

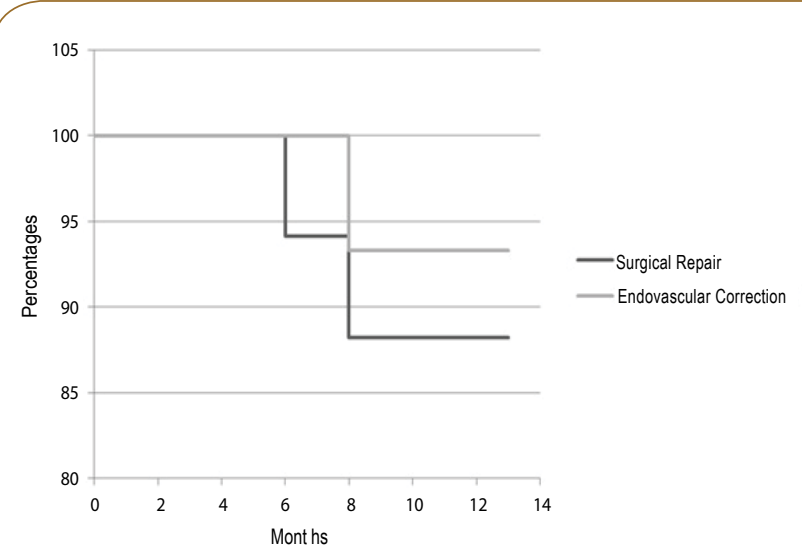

Figure 6 Limb salvage rate. A comparison of limb salvage rate between surgical revision and endovascular correction groups.

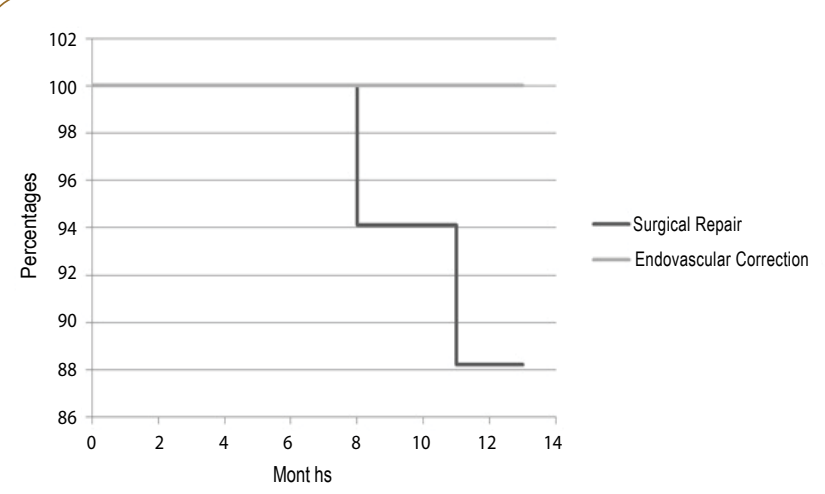

Figure 7 Survival rate: A comparison of survival rate between surgical revision and endovascular correction groups.

of vascular imaging in CLI is to assess the anatomical position, the morphology and the extension of the lesion. The more used first level instrumental examination is the Duplex Ultrasound (DUS), thanks to its non-invasiveness, high reproducibility and low cost. It provides real-time information on the thickness of the arterial wall (intima-media), on location and size of the stenosis/ occlusion, and provides an indirect evaluation of the degree of stenosis through the study of the flow velocity (upstream and downstream of the stenosis). It's also useful to determine the actual need of surgery and to orientate the choice between classic, endovascular or hybrid intervention [17-19]. CT-Angiography or MRI can be used to complete the ultrasound study by providing a more detailed description of the vascular lesion and of the whole arterial tree, in anticipation of a reconstructive surgery or an endovascular approach [20-22]. Digital Subtraction Angiography (DSA), that in the past has been the gold standard in the diagnosis of PAD, has now been replaced by the imaging techniques mentioned above because it's burdened with a certain mortality rate $(0.16 \%)$ and morbidity rate $(0.1 \%)$ due to severe reactions to the contrast medium [23-26]. Currently it's a valuable tool used intraoperatively in the endovascular approach for a more accurate assessment of the level of stenosis and of collateral circulation, and to assess the outcome of the recanalization at the end of the procedure.

The degree of vascular axis involvement is able to significantly affect the choice to perform a revascularization and the modality with which perform it. Despite the recommendations of the TASC II (Table 1), in recent years there has been an increase in the use of primary endovascular correction outside of the traditional indications, except for disease limited to the femoral bifurcation (where the common femoral artery endarterectomy presents patency rates at 5 years of 91-93\%) [27-29], although the data in the literature show the superiority of surgical techniques in terms of 5 years patency rates. This increasing use is commonly justified by the little invasiveness, the low peri-procedural morbidity and mortality rates and the easy repeatability of endovascular correction, and especially from the consideration that if the endovascular revascularization is performed preserving the landing-zones, this doesn't foreclose the possibility of a subsequent bypass surgery.

There are also some clinical conditions of the patient to consider. If on one hand the surgical revascularization guarantees long term patency rates better than that of endovascular treatment (burdened by high percentage of restenosis), on the other hand the endovascular treatment does not require general anesthesia and can be performed with small drawbacks in patients with heart disease, renal failure or with an high surgical and anesthetic risk, and therefore is feasible even in patients who cannot be candidates for surgical bypass because of heavy comorbidity, reduced life expectancy, involvement of the possible sites of distal

Table 1: Recommendations to treatment of femoral popliteal lesions (TASC II) [3].

TASC A and D lesions: Endovascular therapy is the treatment of choice for type $A$ lesions and surgery is the treatment of choice for type $D$ lesions [C].

TASC B and C lesions: Endovascular treatment is the preferred treatment for type $B$ lesions and surgery is the preferred treatment for good-risk patients with type Clesions. The patient's co-morbidities, fully informed patient preference and the local operator's long-term success rates must be considered when making treatment recommendations for type $\mathrm{B}$ and type $\mathrm{C}$ lesions [C]. 
anastomosis in the suffering tissue, unavailability of suitable veins or absence of adequate "landing zone".

Another Achilles Heel is represented from infection of the graft. According to the Centers for Disease Control (CDC) National Nosocomial Infections Surveillance System, vascular and endovascular interventions are clean procedures (risk index categories 1 and 2). Overall incidence of Vascular Surgical Site Infections (VSSI) should range between $2 \%$ and $6 \%$. The reported incidence of infection involving synthetic vascular grafts is around $2 \%$, occurring after 0.2 to $6 \%$ of interventions [30-33]. This variability can be partially explained by differences in duration of post-operative follow-up, type of graft material and method of construction, use of antibiotic prophylaxis, and virulence of the infecting pathogens. Despite aggressive antibiotic administration and surgical treatment, overall mortality rates remain between $10 \%$ and $50 \%$ and overall amputation rate between $15 \%$ and $60 \%$ [34]. Femoro-popliteal graft infections have a lower mortality $(10-25 \%)[35,36]$. Still, the amputation rate approaches $80 \%$ in some studies, especially when infected graft present with sepsis or anastomotic bleeding [37]. Infections are most common in grafts implanted in the inguinal region or in superficial locations, possibly associated with increased bacterial colonization and contamination with the patient's skin flora at this site. Moreover the incidence of graft infection may significantly increase to 2-3 times in case of redo-surgery [38]. For these reasons we believe that the endovascular correction is safer, reducing the manipulation of the graft, especially if it is to be involved the proximal anastomosis, requiring a surgical access in the groin.
Final consideration is the continuous and rapid development by industry of new devices (low profile and very long balloons, drugeluting balloons, directional atherectomy platforms, medicated and non-medicated stents, covered stents, etc.) that make the endovascular approach more feasible even in situations of extreme illness and especially ensuring better patency of the treated vessels.

In order to perform an endovascular correction, is essential the rapid availability of adequate devices. The success rates of this method are also affected by the rapidity of the surgical procedure and particularly from the experience of the operators. The feasibility of this treatment it's therefore a prerogative of high-volume centers with dedicated experts and with a wide range of suitable devices. A professional figure such as that of the vascular surgeon is essential to choose the correct type of surgical approach as it is the only one with the ability to perform all kinds of procedure.

\section{Conclusion}

This topic underlines that endovascular correction after peripheral bypass bleeding is an attractive alternative to surgery, but there are still few data to change the gold standard treatment. The heterogeneity of bypass morphology and bleeding site seems to play a major role in the different outcomes after endovascular correction, and, at the moment, there's not enough evidence from literature to appropriately identify anatomical criteria and the optimal candidate. Randomized Clinical Trials comparing the two treatment options are warranted. 


\section{Reference}

1 [No authors listed] (1991) Second European Consensus Document on chronic critical leg ischemia. Circulation 84: 1-26.

2 Norgren L, Hiatt WR, Dormandy JA (2007) Inter-society consensus for the management of peripheral arterial disease (TASC II). Eur J Vasc Endovasc Surg 45: S5-S67.

3 Dormandy JA, Rutherford RB (2000) Management of peripheral arterial disease (PAD), TASC Working Group, TransAtlantic InterSociety Consensus (TASC). J Vasc Surg 31: S1-S296.

4 Thompson MM, Sayers RD, Varty K (1993) Chronic critical leg ischaemia must be redefined. Eur J Vasc Surg 7: 420-426.

5 Hirsch AT, Haskal ZJ, Hertzer NR (2006) ACC/AHA 2005 guidelines for the management of patients with peripheral arterial disease (lower extremity, renal, mesenteric, and abdominal aortic): executive summary a collaborative report from the American Association for Vascular Surgery/Society for Vascular Surgery, Society for Cardiovascular Angiography and Interventions, Society for Vascular Medicine and Biology, Society of Interventional Radiology, and the ACC/AHA Task Force on Practice Guidelines (Writing Committee to Develop Guidelines for the Management of Patients With Peripheral Arterial Disease) endorsed by the American Association of Cardiovascular and Pulmonary Rehabilitation; National Heart, Lung, and Blood Institute; Society for Vascular Nursing; TransAtlantic InterSociety Consensus; and Vascular Disease Foundation. Circulation 113: e463-e654

6 Greenland P, Abrams J, Aurigemma GP (2000) Prevention Conference $\mathrm{V}$ : Beyond secondary prevention: identifying the high-risk patient for primary prevention: noninvasive tests of atherosclerotic burden: Writing Group III. Circulation 101: E16-E22.

7 American Diabetes Association (2003) Peripheral arterial disease in people with diabetes. ADA Diabetes Care 26: 3333-3341.

8 Al-Qaisi M, Nott DM, King DH (2009) Ankle brachial pressure index (ABPI): An update for practitioners. Vasc Health Risk Manag 5: 833-841.

9 Nicolai SP, Kruidenier LM, Rouwet EV (2009) Ankle brachial index measurement in primary care: are we doing it right? Br J Gen Pract 59: $422-427$

10 Potier L, Abi Khalil C, Mohammedi K (2011) Use and utility of ankle brachial index in patients with diabetes. Eur J Vasc Endovasc Surg 41: 110-116.

11 Andersen CA (2010) Noninvasive assessment of lower extremity hemodynamics in individuals with diabetes mellitus. J Vasc Surg 52 S76-S80.

12 Gornik HL (2009) Rethinking the morbidity of peripheral arterial disease and the "normal" ankle-brachial index. J Am Coll Cardiol 53: 1063-1064.

13 McDermott MM, Liu K, Criqui MH (2005) Ankle-brachial index and subclinical cardiac and carotid disease: the multi-ethnic study of atherosclerosis. Am J Epidemiol 162: 33-41.

14 Clairotte C, Retout S, Potier L (2009) Automated ankle-brachial pressure index measurement by clinical staff for peripheral arterial disease diagnosis in non-diabetic and diabetic patients. Diabetes Care 32: 1231-1236.

15 Holland T (2002) Utilizing the ankle brachial index in clinical practice. Ostomy Wound Manag 48: 38-49.

16 Xu D, Li J, Zou L (2010) Sensitivity and specificity of the anklebrachial index to diagnose peripheral artery disease: a structured review. Vasc Med 15: 361-369.
17 Lowery AJ, Hynes N, Manning BJ (2007) A prospective feasibility study of duplex ultrasound arterial mapping, digital-subtraction angiography, and magnetic resonance angiography in management of critical lower limb ischemia by endovascular revascularization. Ann Vasc Surg 21: 443-451.

18 Edwards JM, Coldwell DM, Goldman ML (1991) The role of duplex scanning in the selection of patients for transluminal angioplasty. J Vasc Surg 13: 69-74.

19 van der Heijden FH, Legemate DA, van Leeuwen MS (1993) Value of Duplex scanning in the selection of patients for percutaneous transluminal angioplasty. Eur J Vasc Surg 7: 71-76.

20 Met R, Bipat S, Legemate DA (2009) Diagnostic performance of computed tomography angiography in peripheral arterial disease: a systematic review and metaanalysis. JAMA 301: 415-424.

21 Levy EM, Viscoli CM, Horwitz RI (1996) The effect of acute renal failure on mortality. A cohort analysis. JAMA 275: 1489-1494.

22 Nelemans PJ, Leiner T, de Vet HC (2000) Peripheral arterial disease: meta-analysis of the diagnostic performance of MR angiography. Radiology 217: 105-114.

23 Hessel SJ, Adams DF, Abrams HL (1981) Complications of angiography. Radiology 138: 273-281.

24 Waugh JR, Sacharias N (1992) Arteriographic complications in the DSA era. Radiology 182: 243-246.

25 Aspelin P, Aubry P, Fransson SG (2003) Nephrotoxic effects in highrisk patients undergoing angiography. N Engl J Med 348: 491-499.

26 Isenbarger DW, Kent SM, O'Malley PG (2003) Meta-analysis of randomized clinical trials on the usefulness of acetylcysteine for prevention of contrast nephropathy. Am J Cardiol 92: 1454-1458.

27 Kang JL, Patel VI, Conrad MF (2008) Common femoral artery occlusive disease: contemporary results following surgical endarterectomy. J Vasc Surg 48: 872-877.

28 Schrijver AM, Moll FL, De Vries JP (2010) Hybrid procedures for peripheral obstructive disease. J Cardiovasc Surg 51: 833-843.

29 Ballotta E, Gruppo M, Mazzalai F (2010) Common femoral artery endarterectomy for occlusive disease: an 8-year single center prospective study. Surgery 147: 268-274.

30 Veith FJ (1979) Surgery of the infected aortic graft. NY: Grune \& Stratton, New York, pp: 532-533.

31 Calligaro KD, Veith FJ (1991) Diagnosis and management of infected prosthetic aortic grafts. Surgery 110: 805-813.

32 Goldstone J, Moore WS (1974) Infection in vascular prostheses: clinical manifestations and surgical management. Am J Surg 128 225-233.

33 Jamieson GG, De Weese JA, Rob CG (1975) Infected arterial grafts. Ann Surg 181: 850-852

34 Edwards WH, Martin RS, Jenkins JM (1987) Primary graft infections. Vasc Surg 6: 235-239.

35 Bunt TJ (1983) Synthetic vascular graft infections I: graft infections. Surg 93: 733-746.

36 Piano G (1995) Infections in lower extremity vascular grafts. Surg Clin North Am 75: 799-809.

37 Brien T, Collin J (1992) Prosthetic vascular graft infection. Br J Surg 79: 1262-1267.

38 Johnson G, Kempczinski RF, Moore WSS (1988) In Rutherford RB, ed. Vascular Surgery. Saunders, Philadelphia, USA, pp: 588-604. 